\title{
Preparation and performance study of hydrotalcites /PLA composites
}

\author{
Liu Hongbo ${ }^{1,2,3}$, Huang Zhiyong ${ }^{1}$ * , Chen Xiuzhu ${ }^{1}$, Chen Yaxin ${ }^{1}$, Lian Haitian ${ }^{1}$ and Li Chong ${ }^{1,2,3}$ \\ ${ }^{1}$ School of Materials Science and Environment, Beijing Institute of Technology Zhuhai, 519088 Zhuhai, China \\ ${ }^{2}$ School of Chemistry and Chemical Engineering, Beijing Institute of Technology, 100081 Beijing, China \\ ${ }^{3}$ Print-rite Unicorn Image Products Co. Ltd. of Zhuhai, 519060 Zhuhai, China
}

\begin{abstract}
D printing technology is known as the core technology of "the third industrial revolution". Poly (lactic acid) (PLA) is a new type of biodegradable thermoplastic resin, which is renewable. It has a wide range of raw materials. It also has the properties of high strength, good biocompatibility, excellent mechanical properties, thermoplastic and transparency. Hydrotalcite (HT) was synthesized by coprecipitation method and modified to prepare the HT/PLA composites. The results show that the structure of HT is good. After KH550 surface modification, a large number of $\mathrm{OH}$ and Si-O groups are introduced into PLA surface. TG results show that the HT sample has good thermal stability. Compared with pure PLA, the glass transition temperature and crystallization temperature of HT / PLA composites decreases, while the melting temperature increases.
\end{abstract}

\section{Introduction}

$3 \mathrm{D}$ printing refers to the technology of layered discrete processing by $3 \mathrm{D}$ printing equipment according to the 3D model files of the product. It accurately stacks certain specific materials layer by layer and rapidly manufactures the required products by laser irradiation. 3D printing is a frontier technology with interdisciplinary [1, 2]. Many technologies such as material technology, information technology, and electromechanical control technology need to be used. Specific structure can be designed according to the model and the precise control of microstructure by computer can be realized. It is known as the core technology of "the third industrial revolution".

At present, 3D printing materials can be divided into two categories: organic polymer materials and inorganic materials. Organic polymer materials are also divided into acrylonitrile styrene butadiene copolymer (ABS), poly (lactic acid) (PLA), polyamide (PA), photosensitive resin and hydrogel. Inorganic materials mainly include metal, ceramics and gypsum. [3]. PLA is a new biodegradable thermoplastic resin, which has renewable properties. Its raw material lactic acid is widely used, which can be obtained by the fermentation of corn, starch and other agricultural products. PLA has high strength and good biocompatibility. Compared with ABS commonly used in FDM process, PLA material is environmentally friendly and has low odor, which is suitable for indoor use. In addition, PLA has excellent mechanical properties, thermoplastic, fiber-forming, transparency and degradation. At the same time, its low shrinkage also makes it impossible to warp even if the hot bed is not heated when printing large-scale model. However, PLA has disadvantages when it is used as bio plastics. The mechanical properties of the material are poor. The toughness and impact resistance are obviously inferior to ABS. And the melt strength is low. Thus it is not suitable to be made too thin and bear load. It is difficult to form and the printing products are brittle. During printing process of unmodified PLA wire, the leakage phenomenon will occur at the nozzle due to the decrease of melt strength. And the rough edge will be formed by sticking on the modelling part, which will affect the surface quality of the printing part [4].

In recent years, a lot of researches have been carried out on toughening and modifying PLA. The researchers toughened and modified PLA by adding inorganic and organic substances into it. Inorganic materials include montmorillonite, carbon nanotubes, talc powder, titanium dioxide, silica, calcium carbonate and HT. A lot of researches show that [5] HT/PLA layered composites have been improved in gas barrier, heat resistance, flame retardant, mechanical properties and electrical properties compared with pure PLA. This will expand the application of PLA in the field of engineering plastics, packaging materials and electronic electrical gas.

HT compounds have layered structure, and their chemical composition can be expressed by the following general formula: $\left[\mathrm{M}^{2+}{ }_{1-\mathrm{x}} \mathrm{M}^{3+}{ }_{\mathrm{x}}(\mathrm{OH})_{2}\right]^{\mathrm{x}+}\left(\mathrm{A}^{\mathrm{n}-}\right)_{\mathrm{x} / \mathrm{n}} \cdot \mathrm{mH}_{2} \mathrm{O}$, where $\mathrm{M}^{2+}$ represents bivalent metal cation; $\mathrm{M}^{3+}$ represents trivalent metal cation, which are located in octahedral voids on the main layer plate; $\mathrm{A}^{\mathrm{n}-}$ is anion, located between layers; $\mathrm{X}$ is charge density. A large class of anionic layered materials with similar structure are formed due to the types of anions and cations as well

$\overline{{ }^{*} \text { Corresponding author: 05066@bitzh.edu.cn }}$ 
as the stoichiometric ratio of divalent and trivalent cations can be adjusted in a large range. The structure of HT is shown in Fig. 1.

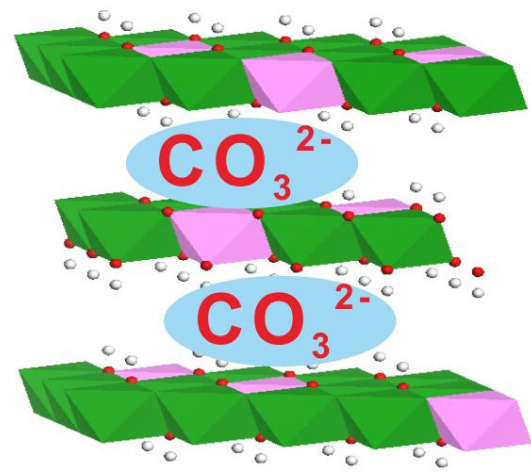

Fig. 1. Structure of HT

In this paper, a series of HT were synthesized by coprecipitation method and modified by KH550. The HT/PLA composites were obtained by melt blending method. The thermal properties of the composites were studied in order to obtain 3D printing materials with excellent performance, so that the PLA materials can obtain better printing effect in practical application.

\section{Experimental}

\subsection{Synthesis of HT}

HT with different metal ratios was synthesized by coprecipitation method. The main synthesis steps are as follows: nitrate solution A with a certain stoichiometric ratio and alkali solution $\mathrm{B}$ with a certain amount of $\mathrm{NaOH}$ and $\mathrm{Na}_{2} \mathrm{CO}_{3}$ were prepared. The salt solution $\mathrm{A}$ was added into the alkali solution $\mathrm{B}$ under high speed stirring, and the $\mathrm{pH}$ value was controlled at about 9. The slurry $\mathrm{C}$ was then transferred to a three-port flask, and stirred vigorously. The mixture was crystallized at $100^{\circ} \mathrm{C}$ for $6 \mathrm{~h}$. The mixture was filtered, washed to neutral with distilled water. The filter cake was dried in a constant temperature drying oven at $70{ }^{\circ} \mathrm{C}$ for $24 \mathrm{~h}$. The obtained solid was ground into powder.

\subsection{Modification of HT}

According to ion exchange method, $12.4 \mathrm{~g} \mathrm{HT}$ and 13.1 $\mathrm{g}$ SDS were dissolved in $640 \mathrm{ml} \mathrm{CO}_{2}$ removal water, and stirred at room temperature for $12 \mathrm{~h}$. The obtained composite was filtered and washed with $\mathrm{CO}_{2}$ removal water until $\mathrm{pH}=7$. $10 \mathrm{~mL} \mathrm{KH550}$ was added into the solution and reacted at $70^{\circ} \mathrm{C}$ for $10 \mathrm{~h}$. The slurry was filtered, dried and ground to obtain the modified HT.

\subsection{Characterization of HT}

The X-ray powder diffractometer (XRD) of Shimadzu $6100 \mathrm{X}$ was used, with $\mathrm{Cu} \mathrm{K} \alpha$ radiation $(\lambda=1.5406 \AA)$, light source wavelength of $\lambda=0.15406 \mathrm{~nm}$, working voltage and current of $40 \mathrm{kV}$ and $30 \mathrm{~mA}$ respectively, scanning speed of $3\left(^{\circ}\right) \cdot \min ^{-1}$, scanning range of $5 \sim$ $75^{\circ} \mathrm{C}$. The infrared absorption spectrum (IR) of the sample was determined by the VECTOR22 Fourier transform infrared spectrometer of Brucker Company in Germany. The $\mathrm{KBr}$ tablet was pressed in the range of $4000 \sim 600 \mathrm{~cm}^{-1}$. The thermal stability of HT samples was characterized by TA company Q5000 thermogravimetric analyser (TGA). $\mathrm{N}_{2}$ was used as carrier gas, the flow rate was $50 \mathrm{ml} \cdot \mathrm{min}^{-1}$, the heating rate was $10{ }^{\circ} \mathrm{C} \cdot \mathrm{min}^{-1}$, and the temperature range was $25 \sim 600{ }^{\circ} \mathrm{C}$.

\subsection{Preparation of HT / PLA composites}

HT/PLA composites were prepared by melt blending method. A certain amount of HT synthesized above was weighed, and was then added to the dried PLA with the amount of $0.5 \%, 1 \%, 2 \%, 4 \%$ and $10 \%$ (by weigh). After evenly mixed, the mixture was then added to the twinscrew extruder for blending. The temperature of each section from the barrel to the head of the extruder was set as $160{ }^{\circ} \mathrm{C}, 180{ }^{\circ} \mathrm{C}, 185{ }^{\circ} \mathrm{C}, 185{ }^{\circ} \mathrm{C}, 180{ }^{\circ} \mathrm{C}$ and $175{ }^{\circ} \mathrm{C}$, and the screw speed was $20 \mathrm{rad} / \mathrm{min}$. After extrusion and granulation, the HT/PLA materials with different HT contents were prepared.

\subsection{Performance test of HT / PLA composites}

Shimadzu DSC-60 differential scanning calorimetry (DSC) was used to characterize the thermal properties of HT/PLA samples. $\mathrm{N}_{2}$ was used as carrier gas, the flow rate was $50 \mathrm{~mL} \cdot \mathrm{min}^{-1}$, the heating rate was $10{ }^{\circ} \mathrm{C} \cdot \mathrm{min}^{-}$ ${ }^{1}$, and the temperature range was $30 \sim 200{ }^{\circ} \mathrm{C}$.

\section{Results and discussions}

\subsection{Structure of HT}

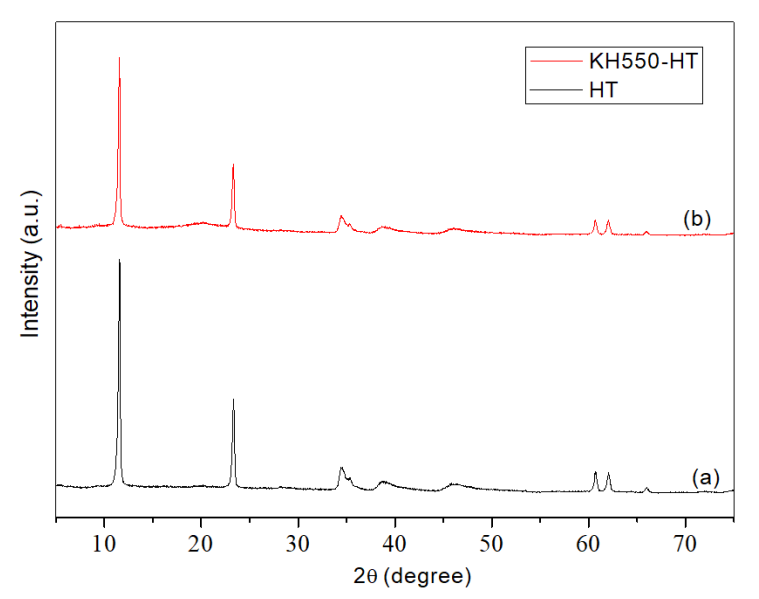

Fig. 2. XRD spectrum of the HT layered materials prepared before and after modification

Fig.2 shows the XRD spectrum of the HT layered materials prepared before and after modification. It can be seen from Fig. 3 that HT shows similar peak patterns. It is found that the peaks are characteristic diffraction peaks of HT by comparing with the standard spectrum. The stable baseline, the sharp peak, and no other 
miscellaneous peaks indicating that the prepared HT layered materials have typical HT structure, good crystalline, single crystal phase and complete crystal phase. It can be concluded that the modified samples still keep the good structure of HT layered materials.

\subsection{Effect of modification}

Fig. 3 shows the IR spectra of HT samples before and after modification. It can be seen from Fig. 3 that the hydrogen oxygen bond stretching vibration peak of $-\mathrm{OH}$ in the layer appeared at about $3428 \mathrm{~cm}^{-1}$ before modification. Compared with the free state $-\mathrm{OH}$ (about $3600 \mathrm{~cm}^{-1}$ ), the peak shifted to the direction of low wave number, indicating that the water molecules between the layers of the sample had hydrogen bond interaction with $\mathrm{CO}_{3}{ }^{2-}$ and hydroxyl groups on the layer. In addition, the splitting peak of asymmetric stretching vibration of $\mathrm{C}-\mathrm{O}$ bond in $\mathrm{CO}_{3}{ }^{2-}$ appears at $1366 \mathrm{~cm}^{-1}$, and it is a symmetrical single peak, which indicates that $\mathrm{CO}_{3}{ }^{2-}$ in the sample layer is well arranged, highly symmetrical and well crystallized. The bending vibration peak of $\mathrm{O}-$ $\mathrm{H}$ in interlayer water appears at $1650 \mathrm{~cm}^{-1}$, and $\mathrm{C}-\mathrm{O}$ stretching vibration peak appears at $792 \mathrm{~cm}^{-1}$. After modification, two sharp peaks appeared at 2842 and $2919 \mathrm{~cm}^{-1}$, were assigned to $-\mathrm{CH}_{3}$ and $-\mathrm{CH}_{2}$ vibration peaks. $-\mathrm{NH}_{2}$ vibration peak appeared at $1467 \mathrm{~cm}^{-1}$. At 1222 and $1134 \mathrm{~cm}^{-1}$ obvious $\mathrm{S}=\mathrm{O}$ vibration peak was appeared. The peak at $990 \mathrm{~cm}^{-1}$ was assigned to $\mathrm{M}-\mathrm{Si}-\mathrm{O}$ stretching vibration, which was in accordance with the literature report [8]. Combined with XRD results, it can be concluded that the surface of HT has been covered by KH550. Therefore, the surface of HT sample was effectively modified.

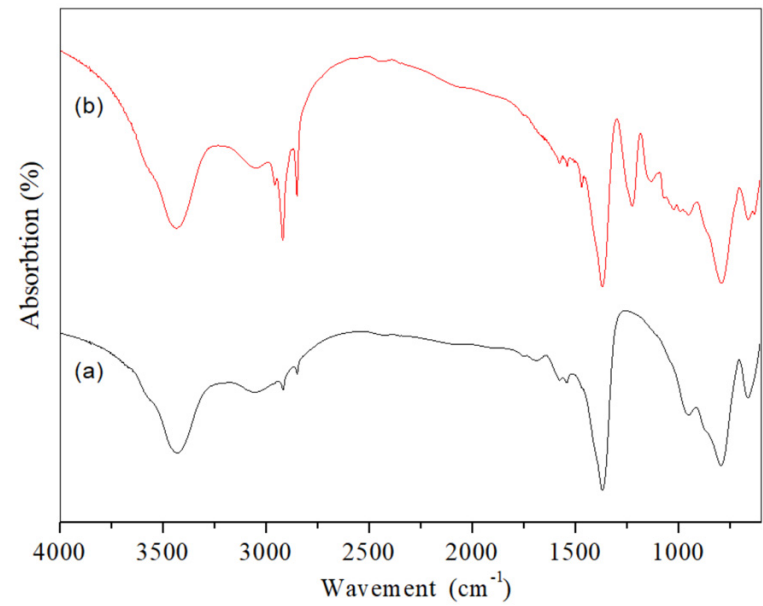

Fig. 3. IR spectra of the HT layered materials prepared before and after modification (a): before modification; (b): after modification

\subsection{Thermo stability of HT}

Fig. 4 shows the TG curve of HT. It can be seen from Fig.4 that the weight loss of HT can be divided into two process. In the first process $\left(<250{ }^{\circ} \mathrm{C}\right)$, HT loses the physically adsorbed water on the crystal surface and the interlayer crystal water. The weight loss rate is about $17 \%$. In the second process $\left(250-500{ }^{\circ} \mathrm{C}\right)$, HT loses the hydroxyl group and some interlayer anions on the laminar, and the total weight loss rate is about 54\%. When the temperature is higher than $500{ }^{\circ} \mathrm{C}$, the decomposition process of HT is basically completed, which is consistent with the results reported in the literature. The relating TGA data of HT was shown in table 1 .

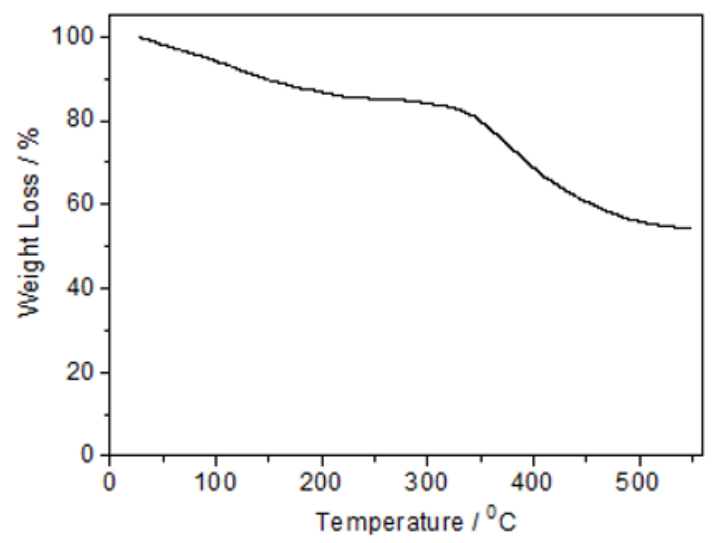

Fig. 4. TG curve of HT

Table 1. TGA data of HT

\begin{tabular}{|c|c|c|}
\hline \multirow{2}{*}{ Sample } & $\begin{array}{c}\text { Temperature } \\
\left({ }^{\circ} \mathrm{C}\right)\end{array}$ & $\begin{array}{c}\text { Weight Loss } \\
(\%)\end{array}$ \\
\hline \multirow{3}{*}{ HT } & $<250$ & 17 \\
\cline { 2 - 3 } & $250 \sim 500$ & 37 \\
\cline { 2 - 3 } & $25 \sim 500$ & 54 \\
\hline
\end{tabular}

\subsection{Effect of HT addition amount}

Fig. 5 shows DSC curves of pure PLA and HT/PLA composites prepared by different HT addition. Table 2 shows the glass transition temperature, crystallization peak temperature and melting peak temperature corresponding to each DSC curve. It can be seen from figure 5 and table 2 that the glass transition temperature and crystallization temperature of HT/PLA composite decrease and the melting temperature increases after the HT/PLA composite is prepared compared with pure PLA.

Table 2 Glass transition temperature, crystallization peak temperature and melting peak temperature of HT/PLA

\begin{tabular}{|c|c|c|c|}
\hline $\begin{array}{c}\text { HT additon } \\
\text { content }\end{array}$ & $\mathrm{T}_{\mathrm{g}} /{ }^{\circ} \mathrm{C}$ & $\mathrm{T}_{\mathrm{C}} /{ }^{\circ} \mathrm{C}$ & $\mathrm{T}_{\mathrm{m}} /{ }^{\circ} \mathrm{C}$ \\
\hline $0 \%$ & 54.23 & 90.06 & 164.98 \\
\hline $0.5 \%$ & 51.15 & 90.04 & 165.58 \\
\hline $1 \%$ & 52.32 & 90.02 & 165.20 \\
\hline $2 \%$ & 51.53 & 89.76 & 165.49 \\
\hline $4 \%$ & 51.83 & 89.84 & 165.14 \\
\hline $10 \%$ & 51.18 & 89.28 & 165.56 \\
\hline
\end{tabular}




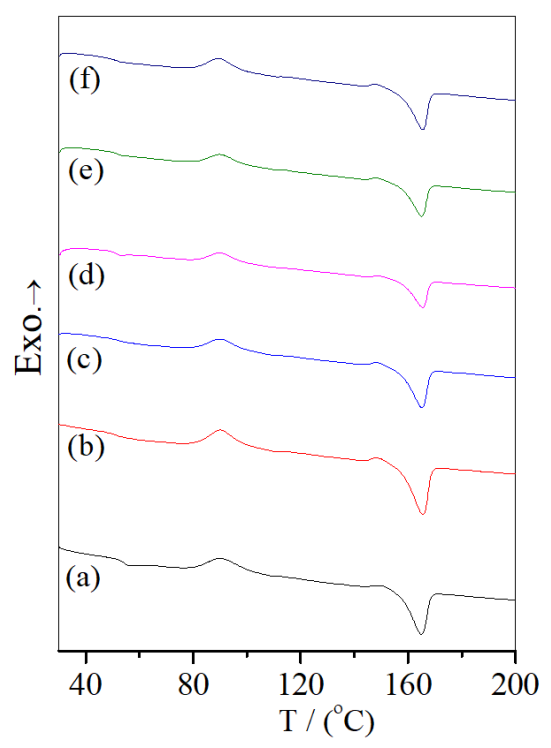

Fig. 5. DSC curves of pure PLA and HT/PLA composites prepared by different HT addition (a): Pure PLA; (b) $0.5 \% \mathrm{HT} / \mathrm{PLA}$; (c): $1 \% \mathrm{HT} / \mathrm{PLA}$; (d): 2\%HT/PLA; (e): 4\%HT/PLA; (f): $10 \%$ HT/PLA

Fig. 6 is the curve of the relationship between the addition amount and the change of temperature. It can be seen from Fig. 6 that in the range of $0.5 \% \sim 10 \%$, the glass transition temperature, crystallization peak temperature and melting peak temperature of HT / PLA composite have no obvious rules with the increase of addition, and the temperature change is higher when the addition is $0.5 \%, 2 \%$ and $10 \%$.

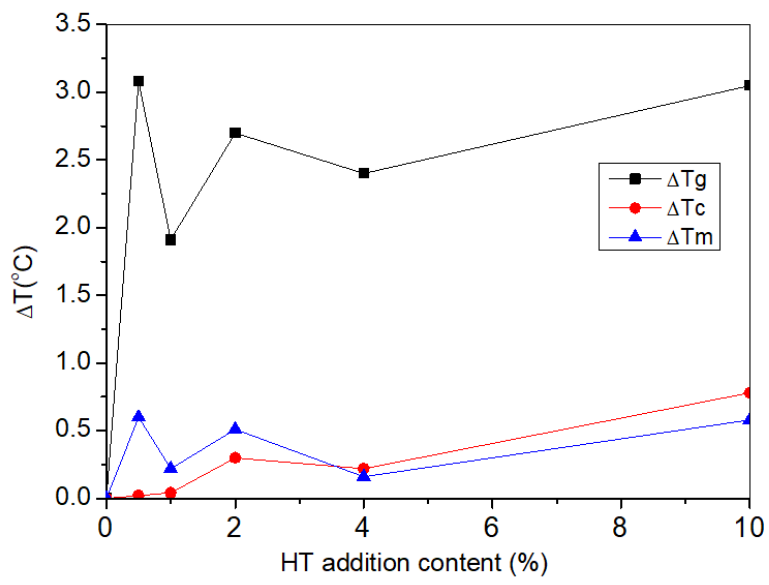

Fig. 6. The curve of the relationship between the addition amount and the change of temperature

\subsection{Effect of HT modification}

Table 3 shows the data of glass transition temperature, crystallization peak temperature and melting peak temperature of HT/PLA and KH-550-HT/PLA. It can be seen from table 3 that the glass transition temperature and melting temperature of HT / PLA composites before modification decrease, while the crystallization temperature increases. After modification the HT/PLA sample has different situation with the one before modification. The temperature is even lower for glass transition temperature and melting temperature, while higher temperature for crystallization peak.

Table 3. Data of glass transition temperature, crystallization peak temperature and melting peak temperature of sample

\begin{tabular}{|c|c|c|c|}
\hline Sample & $\mathrm{T}_{\mathrm{g}} /{ }^{\circ} \mathrm{C}$ & $\mathrm{T}_{\mathrm{C}} /{ }^{\circ} \mathrm{C}$ & $\mathrm{T}_{\mathrm{m}} /{ }^{\circ} \mathrm{C}$ \\
\hline HT/PLA & 52.32 & 90.02 & 165.20 \\
\hline KH550-HT/PLA & 51.45 & 92.51 & 164.86 \\
\hline
\end{tabular}

\section{Conclusions}

A series of HT layered materials were prepared by coprecipitation. The structure and thermal stability of the layered materials were characterized by XRD, IR and TGA. The following conclusions were obtained.

(1) XRD results show that HT layered materials prepared by coprecipitation have typical HT structure, and have good crystalline.

(2) IR results show that the $\mathrm{CO}_{3}{ }^{2-}$ among the layers of HT samples is arranged in order and symmetrical. After the surface modification of KH550, a large number of $-\mathrm{OH}$ and $\mathrm{O}-\mathrm{Si}$ groups are appeared on the PLA surface.

(3) TG results show that the thermal stability of HT samples is good. The weight loss process can be divided into two processes.

(4) DSC results show that the glass transition temperature and crystallization temperature of HT / PLA composites are lower than those of pure PLA, and the melting temperature increases. After modification, HT/PLA has different effect as one before modification.

\section{Acknowledgement}

Authors wishing to acknowledge financial support from Guangdong Province innovation and entrepreneurship training program for college students "Study on properties of poly (lactic acid) modified by inorganic fillers" (2019).

\section{References}

1. S. Zhang, Y. Xu, S. Sun, W. Zang, J. Sun, X. Gu. China plastics. 30, 1(2016)

2. Q. Huang, L. Li, B. Gao. Nation. Defen. Manu. Techno. 50 (2012)

3. Y. Gong, D. Zhang, M. He, W. Song, L. Li, J. Yu. Poly. Mater. Sci. Engin. 34, 6(2018)

4. Y. He. Zhejiang University (2018)

5. Y. Tang, S. Zhang. Guangzhou Chem. Indus. 41, 23(2013)

6. C. Jia. J. Shandong Norm. Univer. 19(2014)

7. Y. Zhang, Y. Zhang, Y. Zhu. Henan Chem. Indus. 24, 12(2007)

8. Z. Ni, X. Fu, etc. Inorg. Chem. 28, 3(2012) 